\title{
SIZE EFFECT ON THE PERMEABILITY AND SHEAR INDUCED FLOW ANISOTROPY OF FRACTAL ROCK FRACTURES
}

\author{
NA HUANG, ${ }^{*, \dagger}$ YUJING JIANG, ${ }^{*, \dagger}$, RICHENG LIU $^{\ddagger}$ and YUXUAN XIA ${ }^{\S}$ \\ ${ }^{*}$ State Key Laboratory of Mining Disaster Prevention and \\ Control Co-founded by Shandong Province and \\ the Ministry of Science and Technology \\ Shandong University of Science and Technology \\ Qingdao 266510, P. R. China \\ ${ }^{\dagger}$ School of Engineering, Nagasaki University \\ 1-14 Bunkyo-machi, 8528521 Nagasaki, Japan \\ ¥State Key Laboratory for Geomechanics and \\ Deep Underground Engineering, China University of \\ Mining and Technology, Xuzhou 221116, P. R. China \\ ${ }^{\S}$ Hubei Subsurface Multi-scale Imaging Key Laboratory \\ Institute of Geophysics and Geomatics \\ China University of Geosciences, Wuhan 430074, P. R. China \\ jiang@nagasaki-u.ac.jp
}

Received September 24, 2017

Accepted November 11, 2017

Published February 15, 2018

\begin{abstract}
The effect of model size on fluid flow through fractal rough fractures under shearing is investigated using a numerical simulation method. The shear behavior of rough fractures with selfaffine properties was described using the analytical model, and the aperture fields with sizes
\end{abstract}

\footnotetext{
TCorresponding author.

This is an Open Access article published by World Scientific Publishing Company. It is distributed under the terms of the Creative Commons Attribution 4.0 (CC-BY) License. Further distribution of this work is permitted, provided the original work is properly cited.
} 
N. Huang et al.

varying from 25 to $200 \mathrm{~mm}$ were extracted under shear displacements up to $20 \mathrm{~mm}$. Fluid flow through fractures in the directions both parallel and perpendicular to the shear directions was simulated by solving the Reynolds equation using a finite element code. The results show that fluid flow tends to converge into a few main flow channels as shear displacement increases, while the shapes of flow channels change significantly as the fracture size increases. As the model size increases, the permeability in the directions both parallel and perpendicular to the shear direction changes significantly first and then tends to move to a stable state. The size effects on the permeability in the direction parallel to the shear direction are more obvious than that in the direction perpendicular to the shear direction, due to the formation of contact ridges and connected channels perpendicular to the shear direction. The variances of the ratio between permeability in both directions become smaller as the model size increases and then this ratio tends to maintain constant after a certain size, with the value mainly ranging from 1.0 to 3.0.

Keywords: Fracture; Size Effect; Permeability; Anisotropy.

\section{INTRODUCTION}

The hydro-mechanical characteristics of single fractures at multiple scales strongly influence the fluid flow through fractured rock masses, the exploitation of unconventional oil and gas reservoirs and the development of nuclear waste repositories. 1-4 Therefore, modeling of flow behavior considering the influences of geometrical and hydraulic properties of fracture continues to be an active area of scientific investigation ${ }^{5 / 7}$ The prerequisites of modeling consist, to a large extent, in determining the complex internal topography of a fracture and its evolution under the mechanical stresses. Thus, various analytical equations, experimental tests and numerical methods have been proposed to quantify the aperture distribution and flow behavior in the fracture under normal and shear stresses. [1]

Single fracture has been approximately modeled by using two smooth parallel plates that are separated by a constant distance, with the fluid flowing through it that follows the cubic law ${ }^{12[13}$ However, in the nature, the surfaces of most rock fractures are rough, which results in nonuniformly distributed aperture fields in fractures. Various methods based on the techniques such as the tomography scanning and calibration of dyed fluid have been proposed to measure the roughness of fracture surface without destroying the initial fracture sample.1415] Correspondingly, the parameters such as joint roughness coefficient (JRC), the root-mean-square of the fracture profile wall slope $\left(Z_{2}\right)$ and fractal dimension $\left(D_{f}\right)$ are defined to quantitatively characterize the surface roughness ${ }^{16}[18$ Laboratory tests and numerical simulations have been performed to classify how the fracture roughness affects the fluid flow paths and the permeability of fractures using these surface roughness parameters. The results show that the rough fracture walls generate the complex and variable aperture fields that induce the tortuous flow paths. Compared with the parallel plate model, the variable apertures can either increase or decrease the permeability, depending on the distributions of flow channels and the contact areas. 19

The rock fractures in the deep underground are generally subjected in situ stresses containing normal and shear stresses. $22[25$ The aperture fields and the flow paths in fractures are changed by both normal stress and shear displacement. The normal stress tends to close the fracture with more contact areas that hinder the flow through the fracture, while the shear displacement tends to open the fracture due to shear dilation and thus increases the permeability of the fracture.

The hydraulic properties of fractured rock masses are usually scale dependent, and the small laboratory samples only represent a fraction of natural fracture characteristics. Bandis et al $\stackrel{26}{[}$ conducted a series of direct shear experiments to analyze the size effects on the shear behavior of rock fractures. They concluded that the significant scale effects exist on both shear strength and deformation response, and the potential sources of the scale effects are mainly attributed to the geometrical and strength characteristics of fracture surface roughness. Giwelli et $a l ! 27$ studied the effect of fracture size on the closure behavior of fractures and the results show that the closure increases with the increasing fracture size. The shear induced aperture field and the flow behavior through the fracture are also scale dependent. Figure 1 shows the fracture samples of 


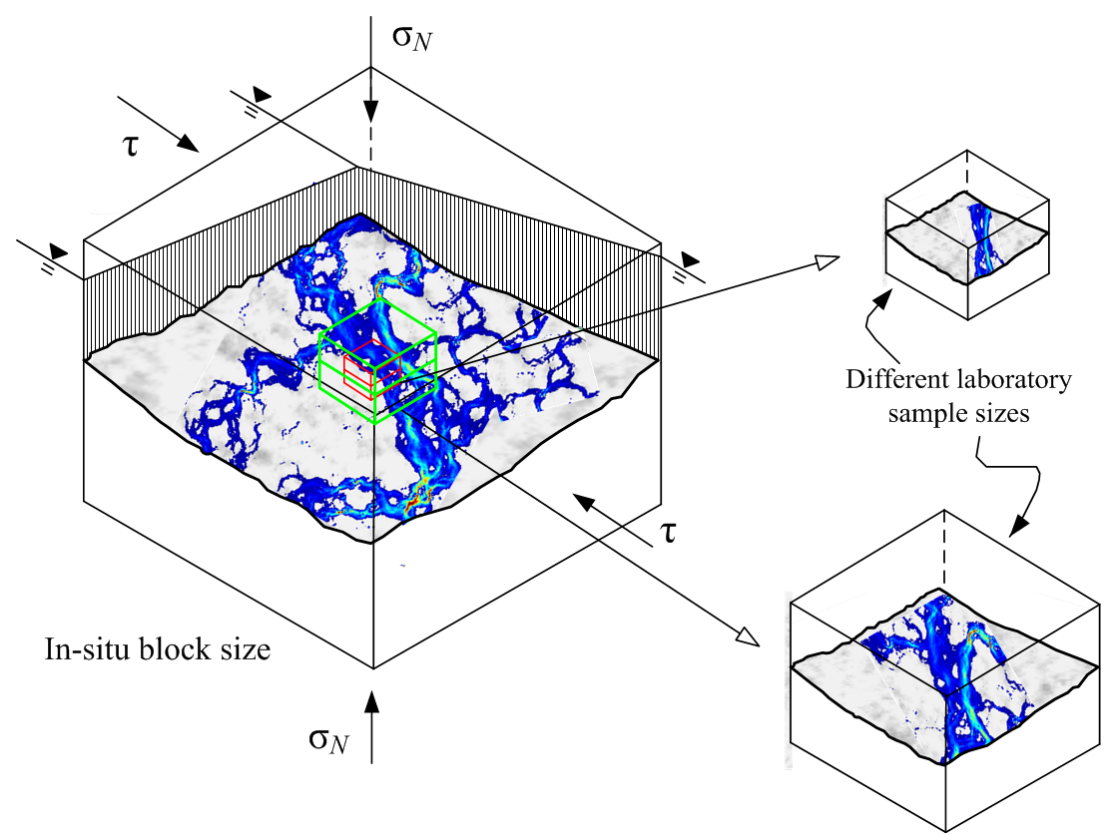

Fig. 1 Different laboratory samples extracted from the in situ fracture with the flow channels indicated.

different laboratory sizes that are extracted from the in situ fractures ${ }^{28}$ The distribution of apertures varies with sample size, which results in different flow channels through the fractures

This study aims to investigate the influences of fracture size on the permeability and the shear induced flow anisotropy of the natural rough-walled rock fractures. A series of fractures with different surface roughness that was represented by JRC and

(a)

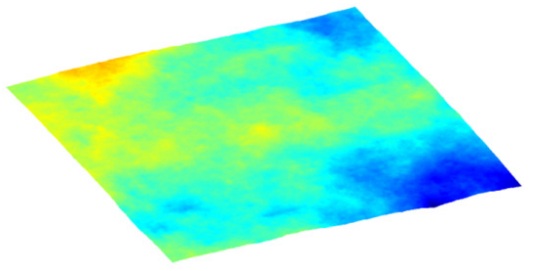

(c)

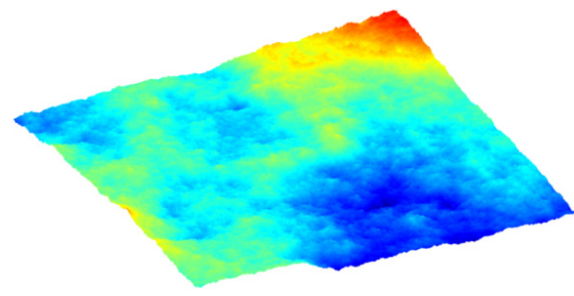

fractal dimension $(D)$ were generated. Shear displacements varying from 3 to $20 \mathrm{~mm}$ were applied to each fracture, and aperture fields of $25-200 \mathrm{~mm}$ in size were extracted from the model at each shear displacement. Fluid flow through the fracture was simulated by solving the Reynolds equation. The aperture variation and flow tortuosity that were resulted from fracture surface roughness and shear displacement were estimated. Influences of fracture

(b)

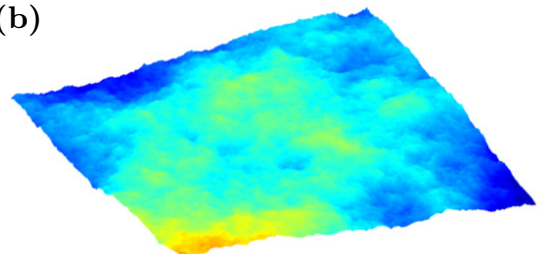

(d)

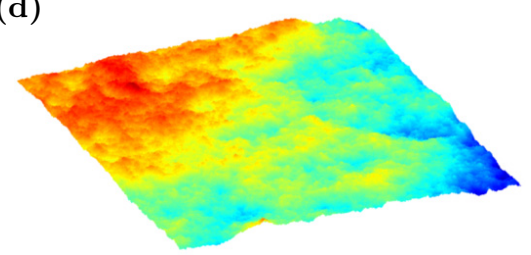

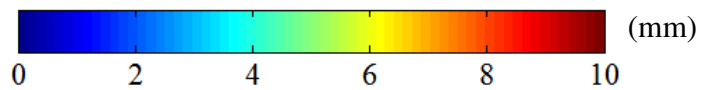

Fig. 2 Fracture surfaces of different roughness generated using the modified SRA method: $(\mathbf{a})$ JRC $=2.49, D_{f}=2.35$; (b) $\mathrm{JRC}=7.36, D_{f}=2.40 ;$ (c) $\mathrm{JRC}=12.38, D_{f}=2.45$ and $(\mathbf{d}) \mathrm{JRC}=17.31, D_{f}=2.50$. 
size on the flow channels, permeability and the flow anisotropy of the fracture were analyzed.

\section{FRACTURE SURFACE PROPERTIES}

The accuracy of quantification of the aperture field for the rough fracture depends, to a large extent, on the model of fracture surface that is usually stochastic and irregular. Fractal geometry that was developed by Mandelbrot et al. ${ }^{29}$ has been widely used to model the irregular objects and fractional surfaces. $.30-34$ The previous studies have shown that the real fracture surfaces can be described by fractal geometry obeying the self-affine scaling fractal law $\stackrel{35136}{3}$ This is of great help in many cases since it provides a numerical method to generate the fracture surface that mimic rough characteristic of natural rock fractures.

The method of self-affine fracture surface generation employed in this study is similar with the earlier works that used Fractional Brownian motion (fBm) to model the surface $\stackrel{37-39}{-3 n}$ 2D fBm, a function $Z(x, y)$ with the independent spatial variables $x$ and $y$ is defined and it is continuous and singlevalued. The stationary increment, $\left[Z\left(x+l_{x}, y+\right.\right.$ $\left.\left.l_{y}\right)-Z(x, y)\right]$, over the distance $l=\sqrt{l_{x}^{2}+l_{y}^{2}}$ displays a Gaussian distribution. The statistical self-affine $\mathrm{fBm}$ increment is expressed as

$$
\begin{gathered}
\left\langle Z\left(x+r l_{x}, y+r l_{y}\right)-Z(x, y)\right\rangle=0, \\
\varepsilon^{2}(r)=r^{2 H} \varepsilon^{2}(1),
\end{gathered}
$$

where $\langle\cdot\rangle$ denotes the mathematical expectation, $r$ is a constant, $\varepsilon^{2}$ is the variance and $H$ is Hurst exponent that is related to the 3D fractal dimension $\left(D_{f}\right)$ by $D_{f}=3-H ![39]$ The modified successive random addition (SRA) algorithm proposed by Liu et al $[40$ is used to generate the related $\mathrm{fBm}$. Different random numbers are used to generate the fracture surface. When these random numbers are fixed, the fracture surface can be obtained repeatedly.

The JRC is proposed by Barton and Choubey 11 to describe the surface roughness of a rock fracture. Based on the height fields of the rough-walled fracture surface, the JRC can be calculated using the following equation that proposed by Tse and Cruden 16 :

$$
\mathrm{JRC}=32.2+32.47 \log \mathrm{Z}_{2},
$$

where $Z_{2}$ is the root-mean-square of the fracture profile wall slope. Figure 2 shows four generated rough fracture surfaces of $256 \mathrm{~mm}$ in size using modified SRA algorithm. The calculated JRC and $D_{f}$ for each fracture surface are shown in Fig. 2, It indicates that a small JRC, which corresponds to a small $D_{f}$ represents a smooth surface with a high spatial correlation, and a large JRC represents a rough surface with many precipitous asperities.

\section{FLOW CALCULATION}

\subsection{Aperture Assignment}

The distribution of fracture aperture defined as the separation distance between two surfaces is one of the most critical factors that determine the hydromechanical property of rough fractures In this study, the two surfaces of a fracture are assumed to be matched at the initial state. The two fracture

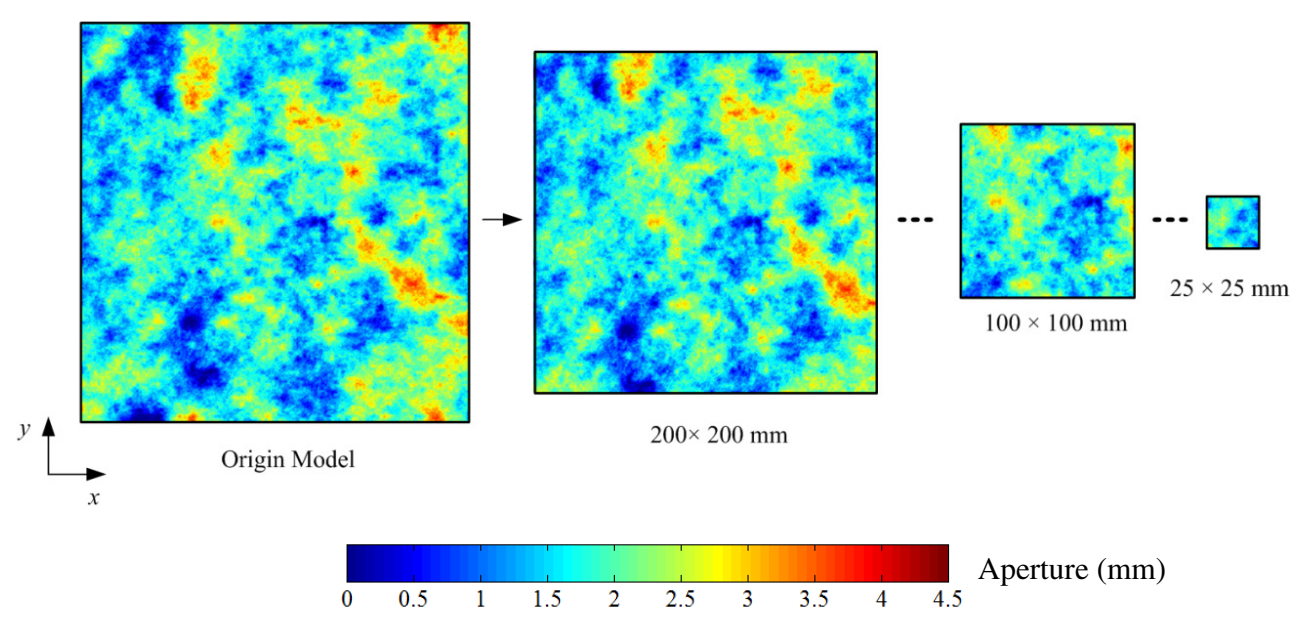

Fig. 3 The extraction of aperture fields of different sizes for fracture with JRC $=17.31$ at $s=20 \mathrm{~mm}$. 
surfaces are initially contacted with the apertures equal to 0 . When a series of shear displacements from 3 to $20 \mathrm{~mm}$ are applied to the fracture, the two fracture surfaces will be shifted and the aperture field is changed with the shear displacement. The aperture distribution at each shear displacement is calculated using the following equation:

$$
b(x, y)=\left\{\begin{array}{rr}
Z(x+s, y) & \\
-Z(x, y)+u & \text { if } Z(x+s, y) \\
& >Z(x, y)-u \\
0 & \text { if } Z(x+s, y) \\
& \leq Z(x, y)-u
\end{array}\right.
$$

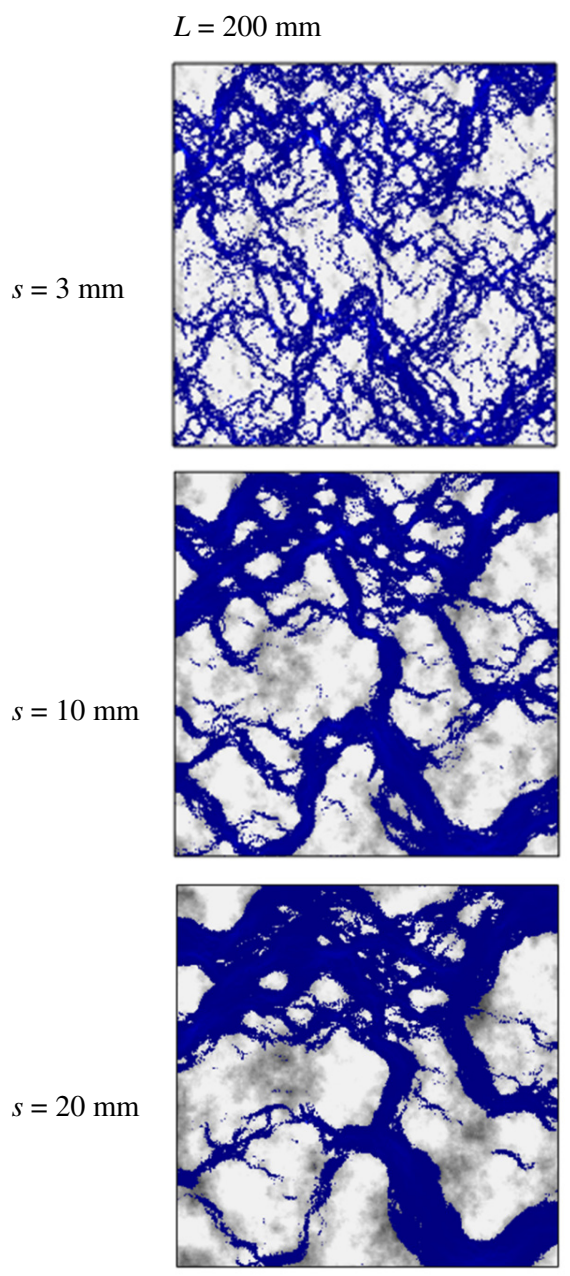

where $s$ is the shear displacement, $u$ is the normal displacement generated at $s$, and it can be determined using the analytical model described by Indraratna et al. ${ }^{42}$ In order to investigate the influence of size scale on the flow behavior, aperture fields with different sizes $L=25,50,75,100,150$ and $200 \mathrm{~mm}$ are extracted from the central part of the initial generated aperture model at each $s$. Figure 3 shows examples of the aperture fields with different sizes extracted from the model at $s=20 \mathrm{~mm}$ for fracture with $\mathrm{JRC}=17.31$.

\subsection{Governing Equation}

At the microscopic scale, the fluid flow through fractures is generally governed by the nonlinear Navier-Stokes equations. However, due to

$L=100 \mathrm{~mm}$

$L=50 \mathrm{~mm}$
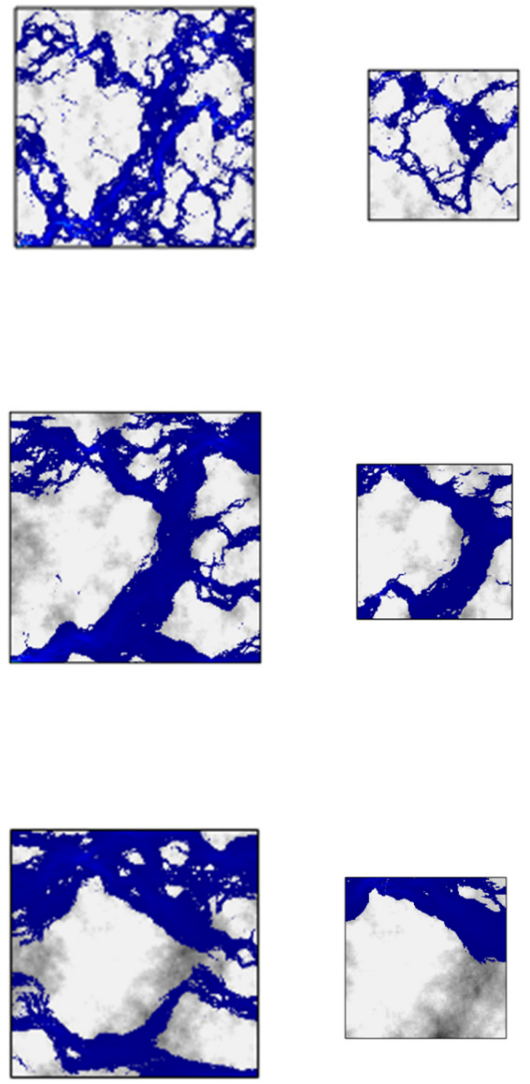

Aperture (mm)

Fig. 4 The aperture distribution and main flow channels for fracture with JRC $=2.49$. The flow paths consist of the elements with the ratios of local flow rate to the total flow rate larger than 0.005 . 
the highly nonlinear system, exact solutions of Navier-Stokes equations are usually very difficult to achieve, especially in the case of natural rough fractures with complex geometry. For a fracture of two perfectly smooth parallel plates, the NavierStokes equations can be simplified to the cubic law, written as 12

$$
Q=-\frac{b^{3}}{12 \mu} \nabla P
$$

However, natural fractures have anisotropic apertures formed by the two rough walls, where the cubic law is obviously over-simplified. When flow velocity is low and the fracture surface does not vary too abruptly, the local cubic law, which is also called as Reynolds equation, can be used to describe the flow in fractures:

$$
\nabla\left(b^{3} \nabla P\right)=0 .
$$

This equation has been utilized to analyze the flow through geological rough fractures $\stackrel{43}{46}$ In this method, the void spaces between the two walls of a fracture are divided into a lot of small simplified parallel-plates, and the aperture of each small plate is calculated based on the Eq. (4). The flow equation is solved using the finite element method (FEM) software COMSOL Multiphysics ${ }^{[38}$ The total flux $Q$ through the fracture equals to the integral of local fluxes through the cross-section of the fracture that can be calculated based on the simulation results. Then, equivalent permeability $K$ of the fracture is

$$
L=100 \mathrm{~mm}
$$

$$
L=50 \mathrm{~mm}
$$
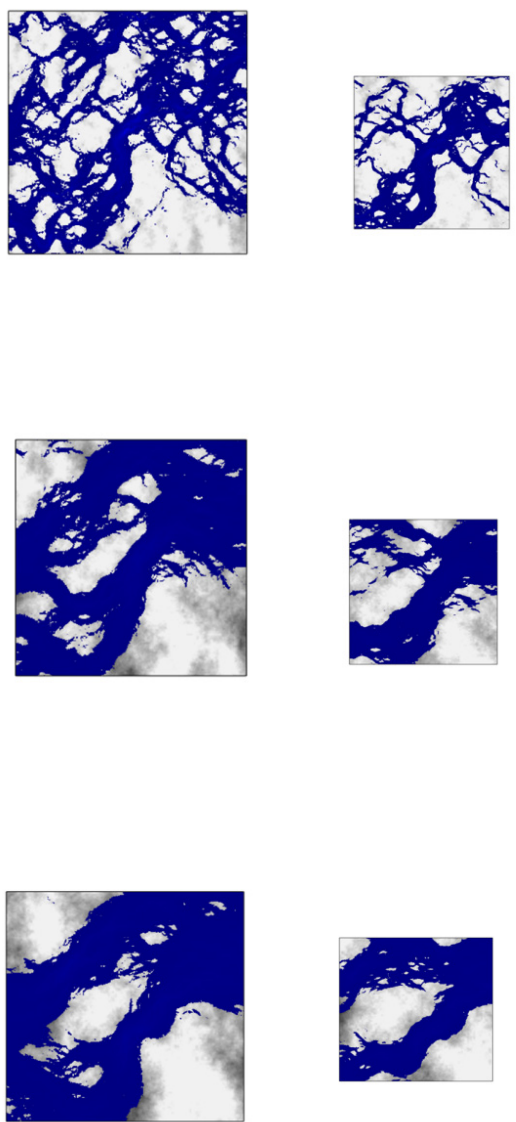

Aperture (mm)

Fig. 5 The aperture distribution and main flow channels for fracture with JRC $=7.36$. The flow paths consist of the elements with the ratios of local flow rate to the total flow rate larger than 0.005 . 
evaluated by

$$
Q=A \frac{K}{\mu} \frac{\Delta P}{L}
$$

where $L$ is the fracture size, $A$ is the cross-sectional area. In order to investigate the flow anisotropy in the rough fracture, two different boundary conditions are considered: unidirectional flow in $x$-direction (parallel to the shear direction) and $y$ direction (perpendicular to the shear direction). For all extracted models with different sizes, a constant hydraulic gradient is maintained between the inlet and out boundaries, and other boundaries are fixed with impervious conditions.

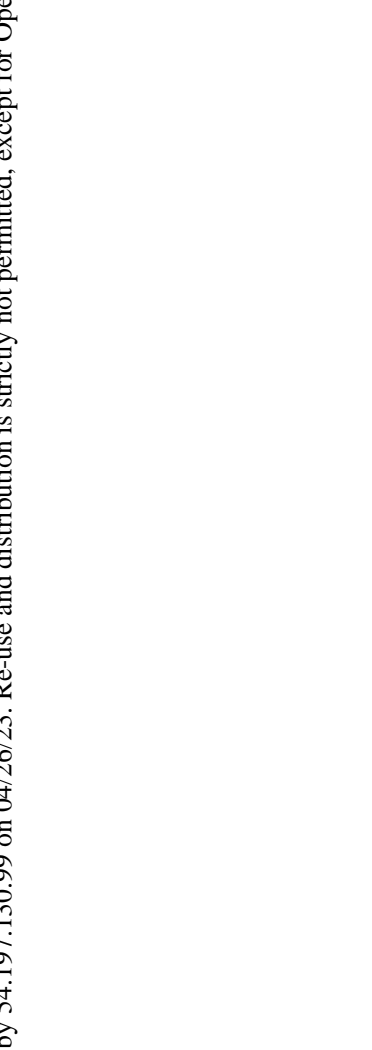

\section{EVOLUTIONS OF APERTURE FIELDS AND FLOW CHANNELS}

The complex flow channels in a fracture have a close relationship with the distribution of fracture apertures. As shear displacement increases, the gradually shifted two fracture rough walls will generate a changed aperture field. Figures 44 7 show the aperture distribution and main flow paths for fractures with different $L$ and JRC under different $s$, where the magnitude of aperture is indicated by the legend and the main flow paths consist of the elements with the ratios of local flow rate to the total flow rate larger than 0.005 . For all fractures, with the

$$
L=100 \mathrm{~mm} \quad L=50 \mathrm{~mm}
$$
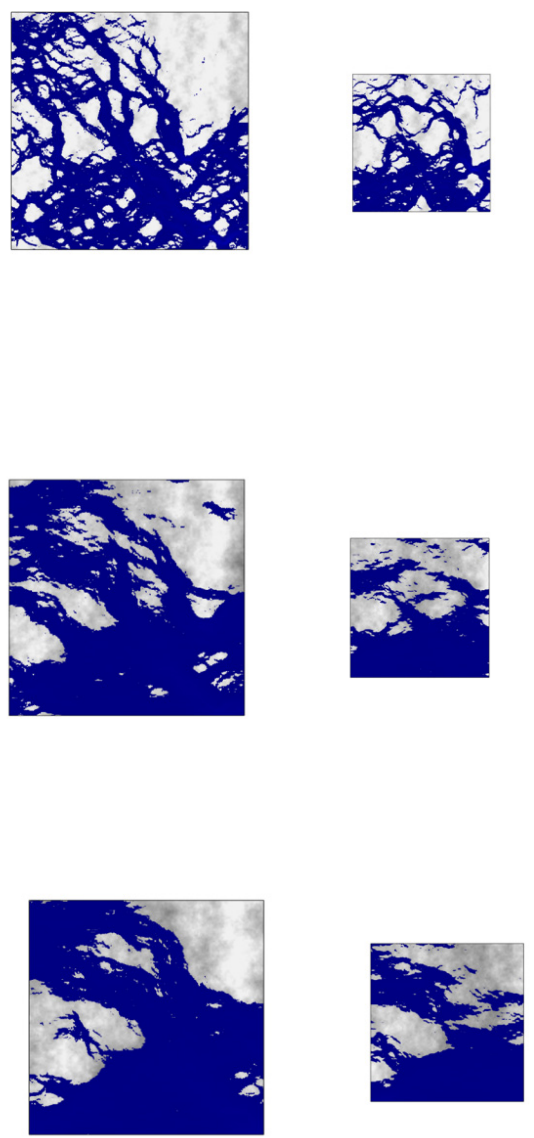

Aperture (mm)

Fig. 6 The aperture distribution and main flow channels for fracture with JRC $=12.38$. The flow paths consist of the elements with the ratios of local flow rate to the total flow rate larger than 0.005 . 
N. Huang et al.

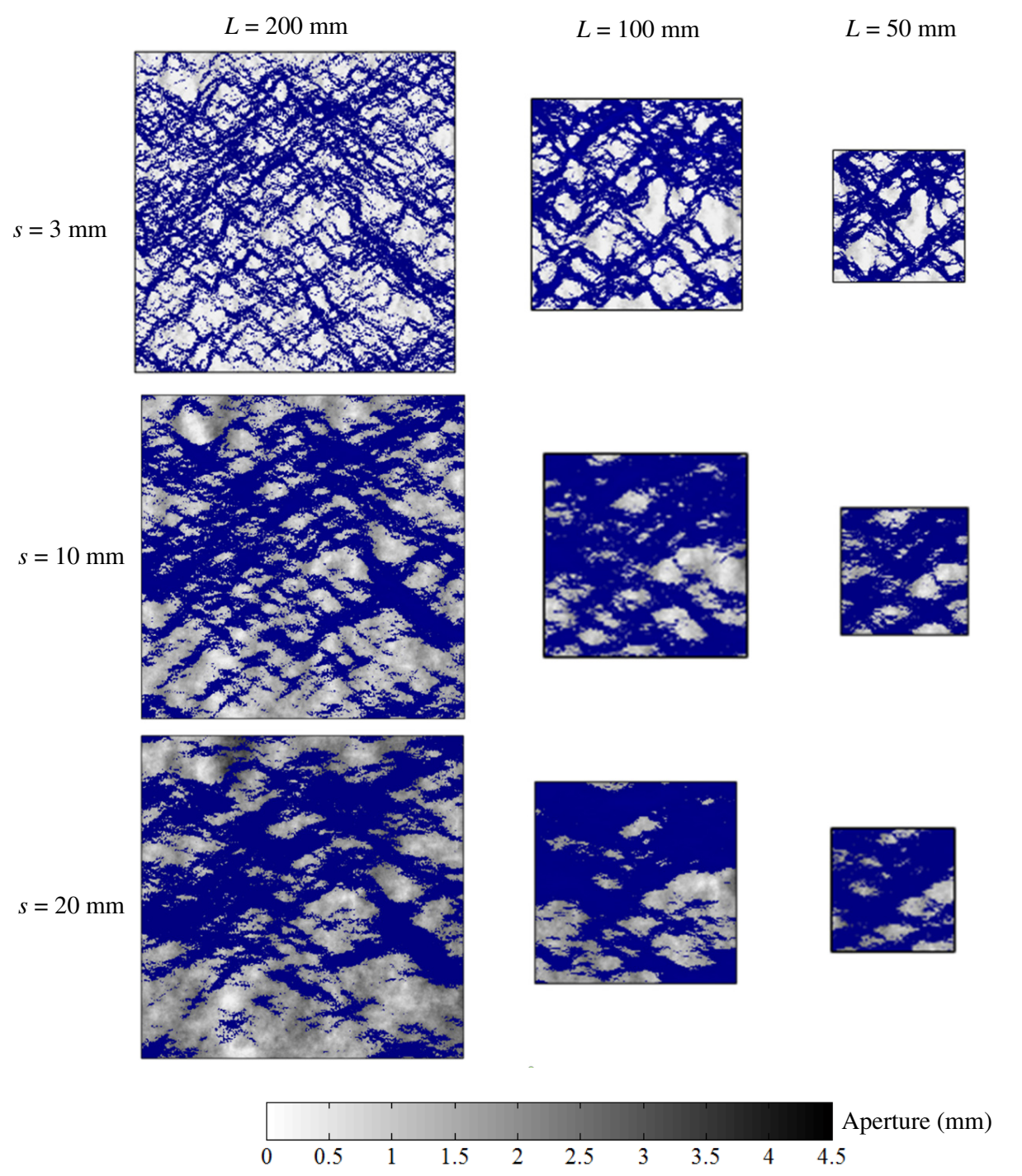

Fig. 7 The aperture distribution and main flow channels for fracture with JRC $=17.31$. The flow paths consist of the elements with the ratios of local flow rate to the total flow rate larger than 0.005 .

increasing of $s$, the apertures increase and the contact areas indicated by the white parts decrease, in spite of the fracture length. This is mainly caused by the shear dilations. ${ }^{4566}$ Comparisons of the aperture distributions of the four fractures at three different $s$ and three different $L$ show that the fracture with a larger JRC generates a larger aperture field.

There exists obvious flow channels for all fractures with different $s$ and different $L$. At a small $s$ of $3 \mathrm{~mm}$, a great number of narrow flow channels exist, bypassing many small pieces of contact areas. The flow paths are distributed almost evenly within the entire fracture plane. As the shear moves on, the flow starts to converge into a few main flow channels, with the dramatic increases of apertures and decreases of contact areas. As $s$ increases to $20 \mathrm{~mm}$, most of the flow concentrates into a few major channels that mainly distributes on the partial areas of fracture plane. Comparisons of the flows of four fractures at three different $s$ and three different $L$ show diversity of flow patterns within the fracture planes. The main flow areas within the fracture plane tend to increase as JRC increases. The flow pattern of fracture with a small size is different with the flow pattern that directly extracted from the model with a large size, which indicates the increase of fracture size alters the effective flow channels through the model. For the fracture with $\mathrm{JRC}=2.49, L=50 \mathrm{~mm}$ and $s=20 \mathrm{~mm}$, there only exists one main flow path between the inlet and outlet boundaries. The fracture with a smaller size is more likely to have a zero aperture field, which results in a totally impermeable model. As fracture size increases, more nonzero aperture areas tend to 
be involved and more alternative flow paths will be available.

\section{EVOLUTION OF PERMEABILITY}

\subsection{Size Effect on the Equivalent Permeability}

The equivalent permeability of each fracture model is calculated and the variations of equivalent permeability $\left(K_{x}\right)$ in direction parallel to the shear direction with $L$ at different $s$ for fractures of different JRC are plotted in Fig. 8. For all fractures, $K_{x}$ increases by almost two orders of magnitudes as the $s$ increases from 3 to $20 \mathrm{~mm}$, which is consistent with the variances of flow channels analyzed in Sec. 4. Comparisons of the $K_{x}$ of the four fractures at the same $s$ show that a rougher fracture has a larger permeability. The variation of $K_{x}$ changes significantly when $L$ is small since the distribution of aperture is almost independent of $L$ for small fracture models. When $L$ increases to a certain value, $K_{x}$ would change slightly so that the variances can be almost negligible. For the fracture with JRC $=2.49, K_{x}$ varies by almost two orders of magnitudes as $L$ changes from 25 to $200 \mathrm{~mm}$, where a sharp decrease at $L=50 \mathrm{~mm}$ occurs. This variance tends to decrease as JRC increases. One possible explanation is that the rougher fracture generates larger apertures over the entire plane and the possibility to have an extreme low permeable area is small.

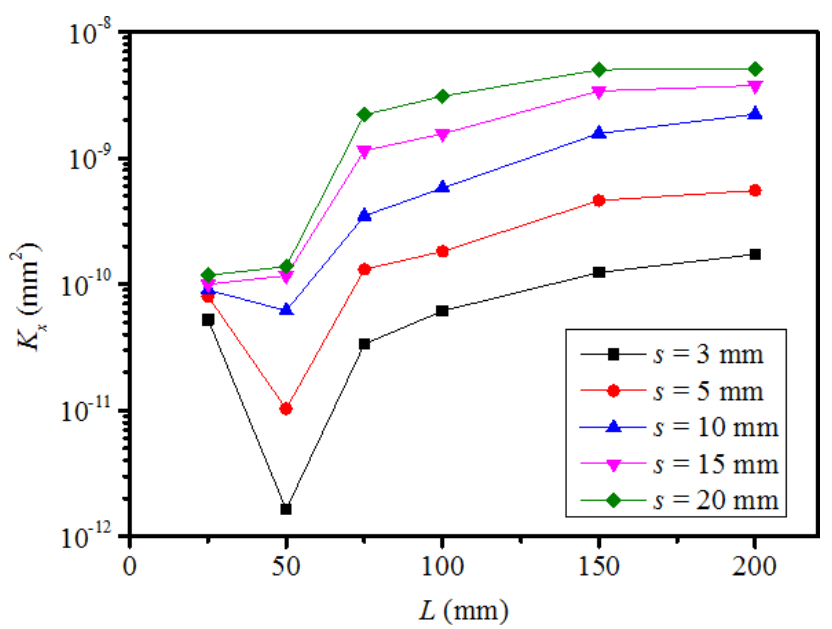

(a)

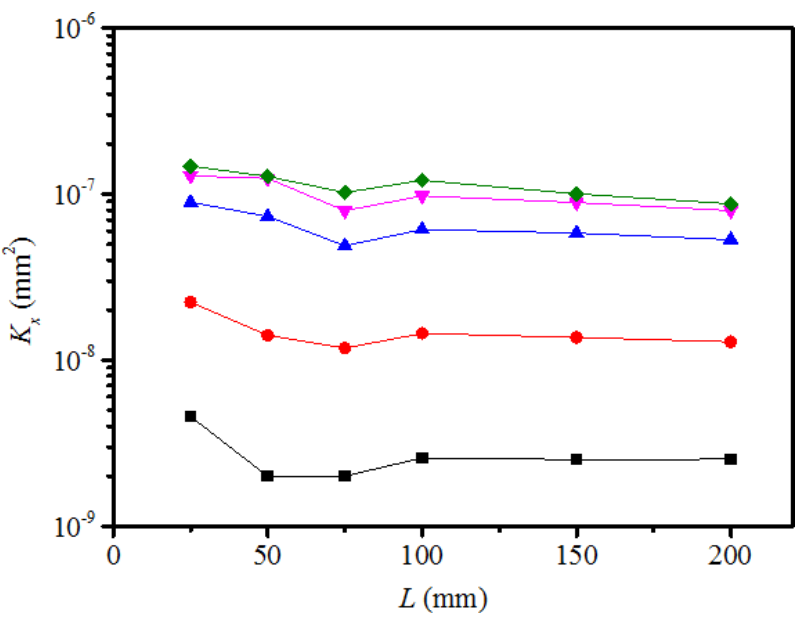

(c)

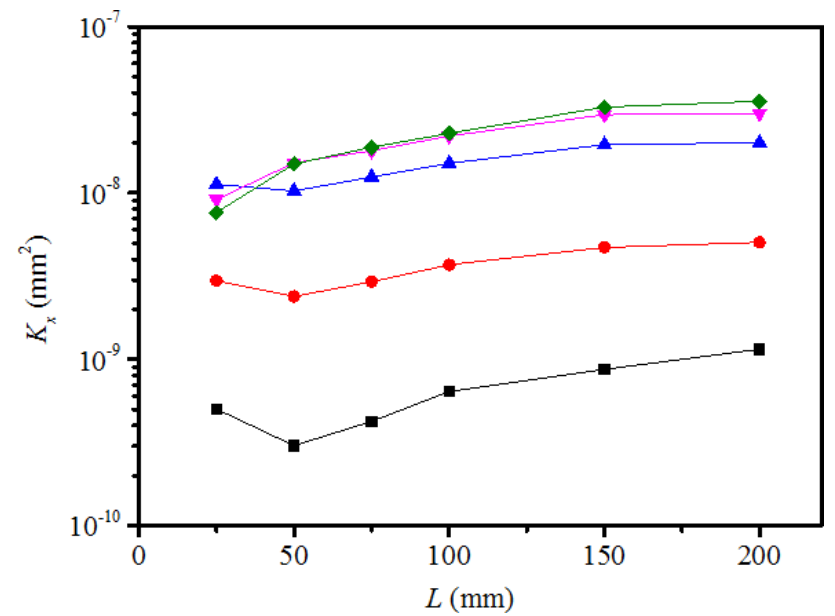

(b)

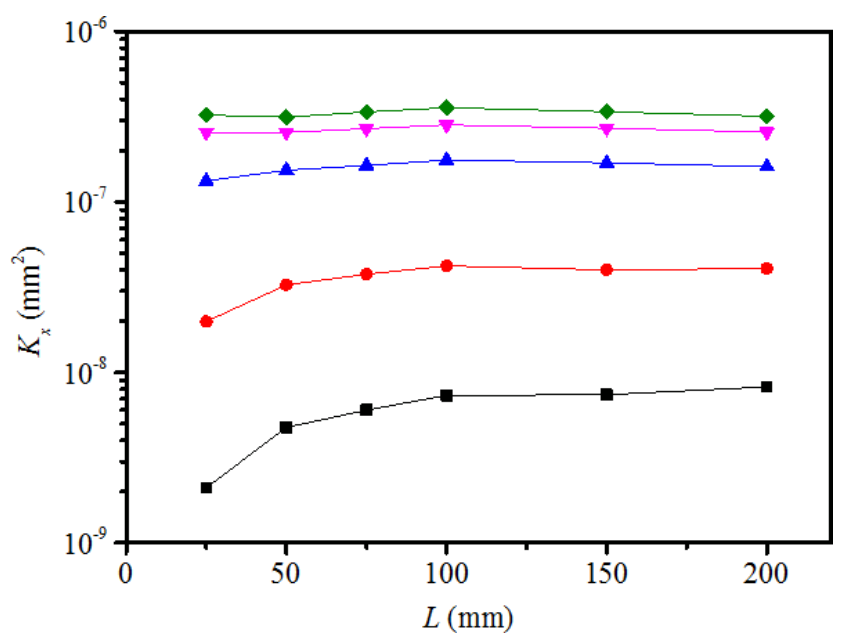

(d)

Fig. 8 Variations of $K_{x}$ with $L$ at different $s$ for fractures of different JRC: $(\mathbf{a})$ JRC $=2.49 ;(\mathbf{b})$ JRC $=7.36 ;(\mathbf{c})$ JRC $=12.38$ and $(\mathbf{d}) \mathrm{JRC}=17.31$. 


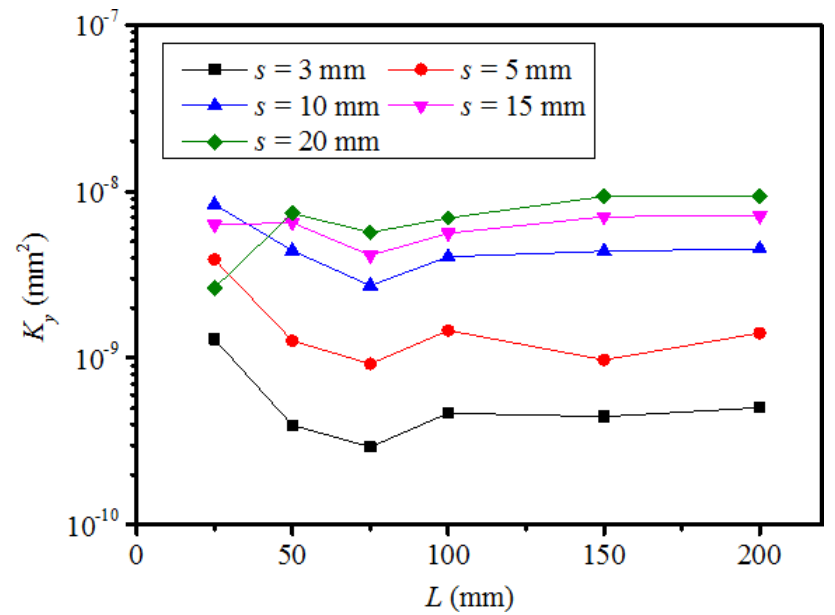

(a)

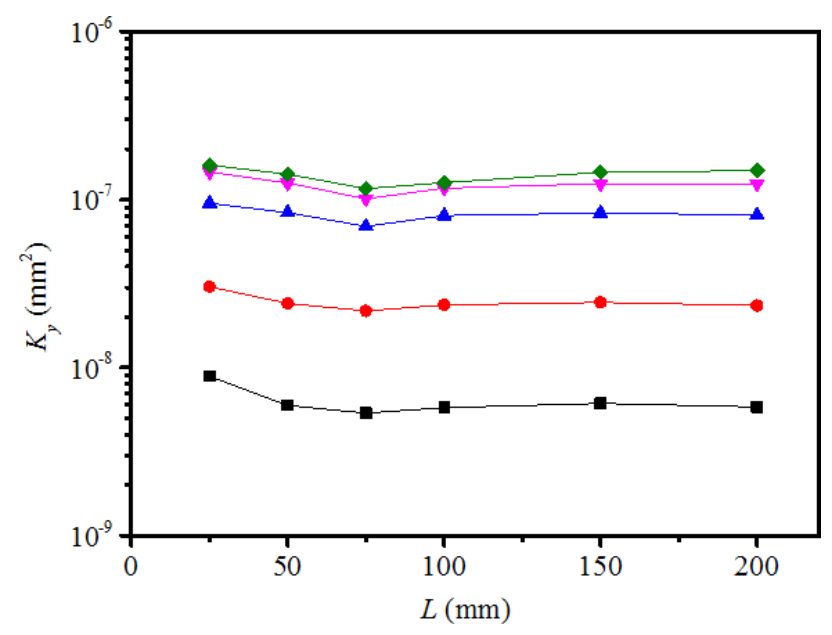

(c)

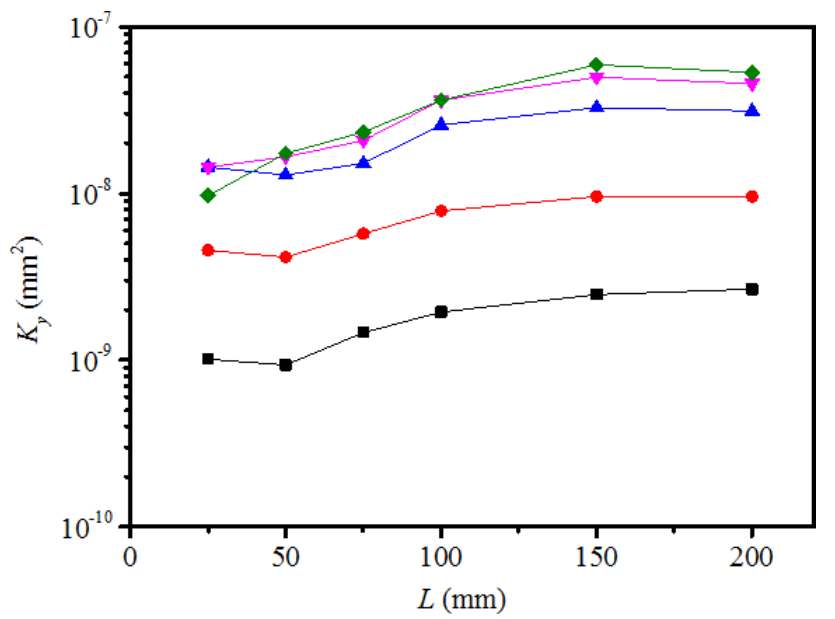

(b)

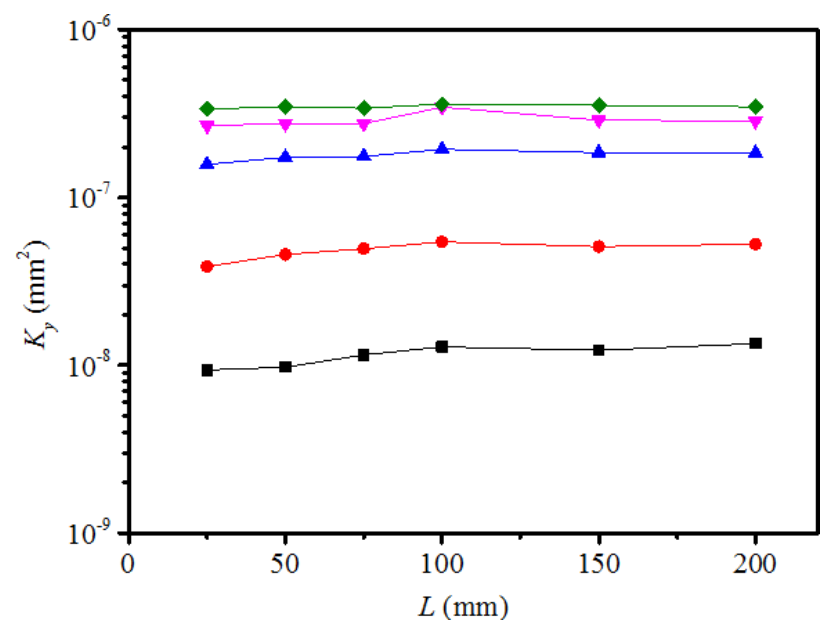

(d)

Fig. 9 Variations of $K_{y}$ with $L$ at different $s$ for fractures of different JRC: $(\mathbf{a})$ JRC $=2.49 ;(\mathbf{b})$ JRC $=7.36 ;(\mathbf{c})$ JRC $=12.38$ and $(\mathbf{d}) \mathrm{JRC}=17.31$.

Figure 9 shows the variations of equivalent permeability $\left(K_{y}\right)$ in direction perpendicular to the shear direction with $L$ at different $s$ for fractures having different JRC, following similar trends with those of $K_{x}$. However, $K_{y}$ is generally larger than $K_{x}$ and the size effects on $K_{y}$ are less obvious than that on $K_{x}$. There may be two reasons: on the one hand, since the two surfaces of the fracture are well matched at the initial state, the upper fracture surface climbs the asperity as the shear displacement increases, which results in the collision between the adjacent fracture asperities and thus generates many stripy ridges of contact perpendicular to the shear direction. These stripy ridges of contacts tend to prevent the flow parallel to the shear direction. On the other hand, the shear-induced void channels tend to be connected along the direction perpendicular to the shear displacement, which generates more effective flow paths perpendicular to the shear direction.

\subsection{Size Effect on the Anisotropy of Permeability}

Previous studies have shown that the permeability of rough fracture shows remarkable anisotropy during shear. $47+50]$ In order to examine the size effect on the anisotropy of permeability, the variations of $K_{y} / K_{x}$ with $L$ at different $s$ for fractures of different JRC are plotted in Fig. 10. The ratio of $K_{y} / K_{x}$ varies remarkably at the small $L$. As $L$ continues to increase, $K_{y} / K_{x}$ tends to move to a relatively stable state with the value mainly ranging from 1.0 to 3.0. For the fractures with $\mathrm{JRC}=2.49, K_{y} / K_{x}$ varies by about two orders of magnitudes as $L$ increases from 25 to $200 \mathrm{~mm}$. But the changes of $K_{y} / K_{x}$ with 


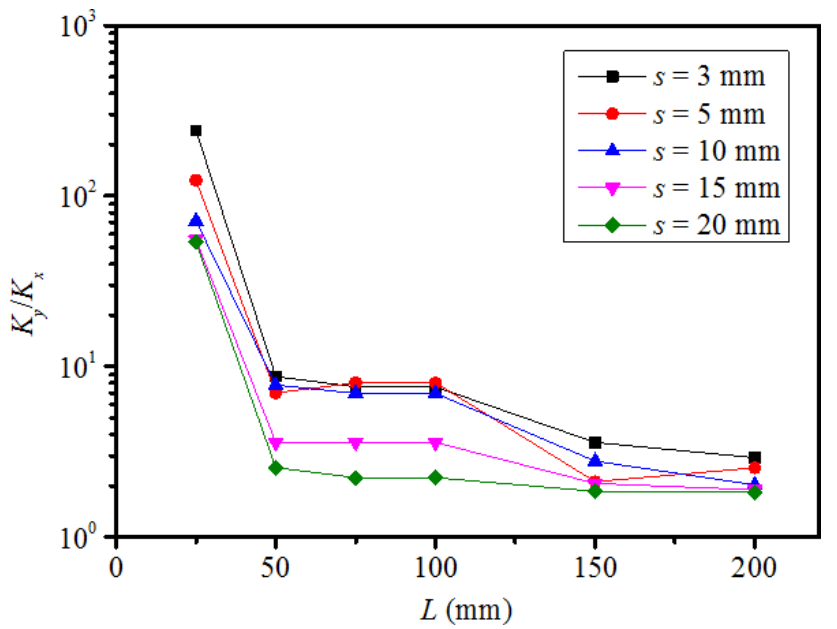

(a)

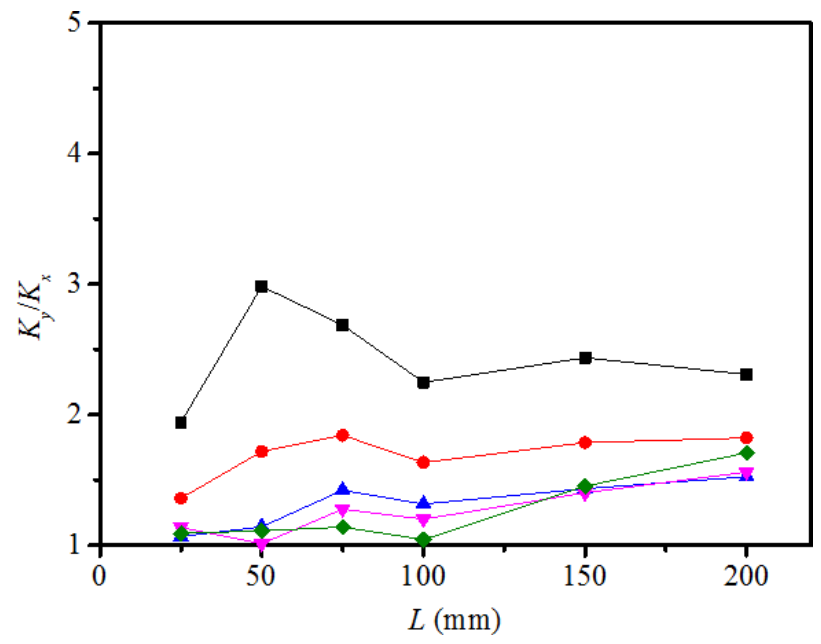

(c)

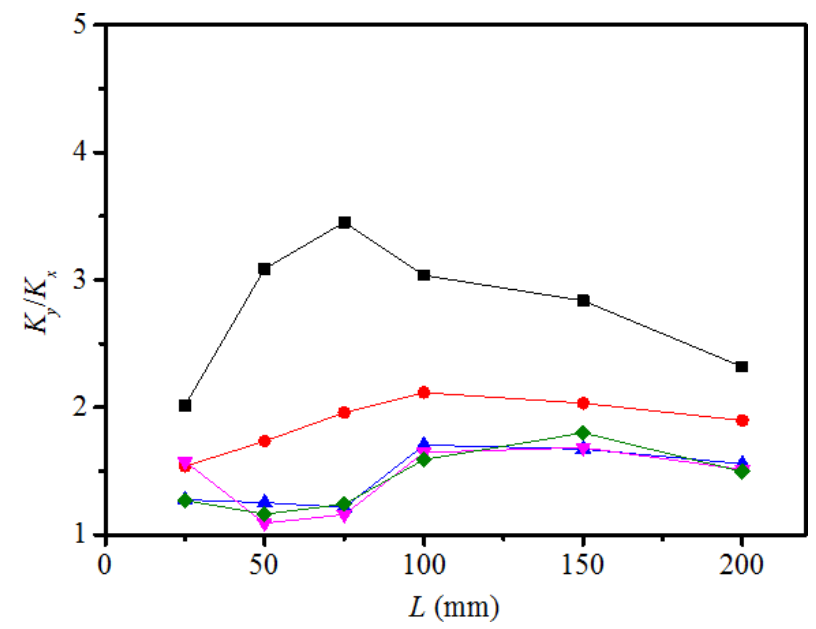

(b)

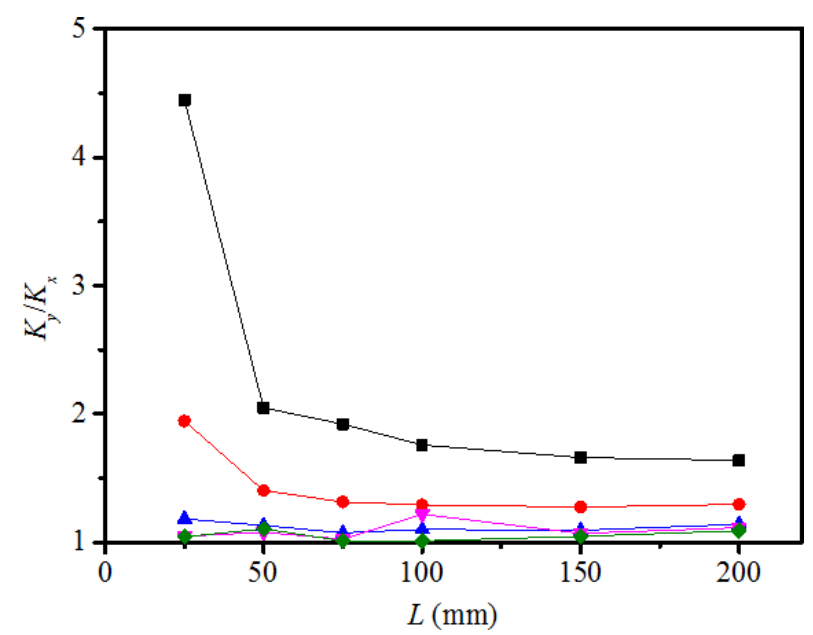

(d)

Fig. 10 Variations of $K_{y} / K_{x}$ with $L$ at different $s$ for fractures of different JRC: (a) JRC $=2.49$; $(\mathbf{b})$ JRC $=7.36$; (c) $\mathrm{JRC}=12.38$ and (d) $\mathrm{JRC}=17.31$.

$L$ for other three fractures with larger JRC become to be less dramatic. This is most likely because of the influences of fracture roughness on the aperture fields that in turn depend on the magnitude of fracture size.

\section{CONCLUSIONS}

In this study, the shear flow tests were simulated on a series of fracture surfaces with self-affine properties. Aperture fields with the size varying from 25 to $200 \mathrm{~mm}$ were extracted under different shear displacements to investigate the size effects on the permeability and shear induced flow anisotropy of rock fractures. Based on the aperture data at each shear interval, fluid flow was simulated by solving Reynolds equation using the FEM software COMSOL Multiphysics. Permeability in the directions both parallel and perpendicular to the shear direction was calculated. Flow channeling and shear induced flow anisotropy in fractures of different sizes under different shear displacements were estimated.

The results show that aperture fields and flow paths in rough rock fractures are influenced by the topography of fracture surface and shear condition. As fracture surface roughness and/or shear displacement increase, the apertures increase and the contact areas decrease in spite of the fracture length, which is mainly caused by the shear dilations for rough fractures. At a small shear displacement, there are a great number of narrow flow 
channels that are indicated by the elements with the ratios of local flow rate to the total flow rate larger than 0.005. As the shear displacement increases, the flow tends to converge into a few main flow channels, with the dramatic increases of apertures and decreases of contact areas. The shapes of flow channels change significantly as the fracture size increases. The permeability in the directions both parallel and perpendicular to the shear direction changes significantly when fracture size is small, and then changes slightly when model size increases to a certain value. The size effects on the permeability in $x$-direction tend to be more obvious than that in $y$-direction. This is mainly caused by the formations of contact ridges and connected channels perpendicular to the shear direction during the shear. The variances of the ratio between permeability in the $x$-direction and that in the $y$-direction become smaller as the model size increases and then this ratio tends to maintain constant after a certain size, with the value mainly ranging from 1.0 to 3.0. In the future, we plan to extend our study to the motion of solute particles in rough fractures, and investigate how the factors including fracture size, roughness and shear displacement affect the solute transport behavior.

\section{ACKNOWLEDGMENTS}

This study has been partially funded by National Natural Science Foundation of China (Grant Nos. 51709260, 51323004, 41572116), Natural Science Foundation of Jiangsu Province, China (No. BK20170276), and JSPS-NSFC Bilateral Joint Research Project and JSPS Grant-in-Aid for Scientific Research (Number 17H03506), Japan. These supports are gratefully acknowledged.

\section{REFERENCES}

1. J. P. Pereira and M. H. De Freitas, Mechanisms of shear failure in artificial fractures of sandstone and their implication for models of hydromechanical coupling, Rock Mech. Rock Eng. 26(3) (1993) 195214.

2. W. Yu and K. Sepehrnoori, Simulation of gas desorption and geomechanics effects for unconventional gas reservoirs, Fuel 116 (2014) 455-464.

3. J. Rutqvist et al., A comparative simulation study of coupled THM processes and their effect on fractured rock permeability around nuclear waste repositories, Environ. Geol. 57(6) (2009) 1347-1360.
4. W. Wei and Y. Xia, Geometrical, fractal and hydraulic properties of fractured reservoirs: A minireview, Adv. Geoenerg. 1(1) (2017) 31-38.

5. K. Bisdom, G. Bertotti and H. M. Nick, The impact of in-situ stress and outcrop-based fracture geometry on hydraulic aperture and upscaled permeability in fractured reservoirs, Tectonophysics 690 (2016) $63-75$.

6. J. Cai et al., Electrical conductivity models in saturated porous media: A review, Earth-Sci. Rev. 171 (2017) 419-433.

7. S. Luo et al., The role of fracture surface roughness in macroscopic fluid flow and heat transfer in fractured rocks, Int. J. Rock Mech. Min. Sci. 87 (2016) 29-38.

8. A. Lavrov, Fracture permeability under normal stress: A fully computational approach, J. Petrol. Explor. Prod. Technol. 7(1) (2017) 181-194.

9. K. Nemoto et al., Direct measurement of contact area and stress dependence of anisotropic flow through rock fracture with heterogeneous aperture distribution, Earth Planet Sci. Lett. 281(1) (2009) 81-87.

10. N. Watanabe, N. Hirano and N. Tsuchiya, Determination of aperture structure and fluid flow in a rock fracture by high-resolution numerical modeling on the basis of a flow-through experiment under confining pressure, Water Resour. Res. 44(6) (2008) W06412.

11. K. Bisdom, G. Bertotti and H. M. Nick, The impact of different aperture distribution models and critical stress criteria on equivalent permeability in fractured rocks, J. Geophys. Res. Solid Earth 121(5) (2016) 4045-4063.

12. P. A. Witherspoon et al., Validity of cubic law for fluid flow in a deformable rock fracture, Water Resour. Res. 16(6) (1980) 1016-1024.

13. R. W. Zimmerman and I. W. Yeo, Fluid flow in rock fractures: From the Navier-Stokes equations to the cubic law, Dyn. Fluids Fract. Rock 122 (2000) 213224.

14. E. Isakov et al., Fluid flow through rough fractures in rocks I: High resolution aperture determinations, Earth Planet Sci. Lett. 191(3) (2001) 267-282.

15. S. P. Bertels, D. A. DiCarlo and M. J. Blunt, Measurement of aperture distribution, capillary pressure, relative permeability, and in situ saturation in a rock fracture using computed tomography scanning, Water Resour. Res. 37(3) (2001) 649-662.

16. R. Tse and D. M. Cruden, Estimating joint roughness coefficients, Int. J. Rock Mech. Min. Sci. Geomech. Abstr. 16(5) (1979) 303-307.

17. D. Crandall, G. Bromhal and Z. T. Karpyn, Numerical simulations examining the relationship between wall-roughness and fluid flow in rock fractures, Int. J. Rock Mech. Min. Sci. 47(5) (2010) 784-796. 
18. E. Bouchaud, G. Lapasset and J. Planes, Fractal dimension of fractured surfaces: A universal value?, Europhys. Lett. 13(1) (1990) 73.

19. Y. Méheust and J. Schmittbuhl, Flow enhancement of a rough fracture, Geophys. Res. Lett. 27(18) (2000) 2989-2992.

20. N. Huang et al., A numerical method for simulating fluid flow through 3-D fracture networks, J. Nat. Gas Sci. Eng. 33 (2016) 1271-1281.

21. L. Moreno et al., Flow and tracer transport in a single fracture: A stochastic model and its relation to some field observations, Water Resour. Res. 24(12) (1988) 2033-2048.

22. B. S. Aadnoy and J. S. Bell, Classification of drillinginduced fractures and their relationship to in-situ stress directions, Log Anal. 39(6) (1998).

23. M. K. Rahman, M. M. Hossain and S. S. Rahman, A shear-dilation-based model for evaluation of hydraulically stimulated naturally fractured reservoirs, Int. J. Numer. Anal. Met. 26(5) (2002) 469497.

24. T. Zhang et al., A new model for calculating permeability of natural fractures in dual-porosity reservoir, Adv. Geo-Energ. Res. 1(2) (2017) 86-92.

25. H. Singh and J. Cai, Screening improved recovery methods in tight-oil formations by injecting and producing through fractures, Int. J. Heat Mass Transf. 116 (2018) 977-993.

26. S. Bandis, A. C. Lumsden and N. R. Barton, Experimental studies of scale effects on the shear behaviour of rock joints, Int. J. Rock Mech. Min. Sci. 18(1) (1981) 1-21.

27. A. A. Giwelli, K. Sakaguchi and K. Matsuki, Experimental study of the effect of fracture size on closure behavior of a tensile fracture under normal stress, Int. J. Rock Mech. Min. Sci. 46(3) (2009) 462-470.

28. T. Koyama et al., Numerical simulation of shearinduced flow anisotropy and scale-dependent aperture and transmissivity evolution of rock fracture replicas, Int. J. Rock Mech. Min. Sci. 43(1) (2006) 89-106.

29. B. B. Mandelbrot, D. E. Passoja and A. J. Paullay, Fractal character of fracture surfaces of metals, Nature 308(5961) (1984) 721-722.

30. J. Cai et al., Fractal characterization of dynamic fracture network extension in porous media, Fractals 25(2) (2017) 1750023.

31. R. Liu et al., A numerical approach for assessing effects of shear on equivalent permeability and nonlinear flow characteristics of 2-D fracture networks, Adv. Water Resour. 111 (2018) 289-300.

32. P. Xu and B. Yu, Developing a new form of permeability and Kozeny-Carman constant for homogeneous porous media by means of fractal geometry, Adv. Water Resour. 31(1) (2008) 74-81.
33. W. Wei, J. Cai, X. Hu and Q. Han, An electrical conductivity model for fractal porous media, Geophys. Res. Lett. 42(12) (2015) 4833-4840.

34. W. Wei et al., A numerical study on fractal dimensions of current streamlines in two-dimensional and three-dimensional pore fractal models of porous media, Fractals 23(1) (2015) 1540012.

35. B. B. Mandelbrot, Self-affine fractals and fractal dimension, Phys Scr. 32(4) (1985) 257.

36. L. Ponson et al., Anisotropic self-affine properties of experimental fracture surfaces, Int. J. Fract. 140 (1-4) (2006) 27-37.

37. Z. Ye et al., Two-phase flow properties of a horizontal fracture: The effect of aperture distribution, $A d v$. Water Resour. 76 (2015) 43-54.

38. N. Huang et al., A predictive model of permeability for fractal-based rough rock fractures during shear, Fractals 25(5) (2017) 1750051.

39. M. Wang et al., Influence of surface roughness on nonlinear flow behaviors in 3D self-affine rough fractures: Lattice Boltzmann simulations, Adv. Water Resour. 96 (2016) 373-388.

40. H. H. Liu et al., A corrected and generalized successive random additions algorithm for simulating fractional Levy motions, Math. Geol. 36(3) (2004) 361-378.

41. N. Barton and V. Choubey, The shear strength of rock joints in theory and practice, Rock Mech. Rock Eng. 10(1) (1977) 1-54.

42. B. Indraratna et al., Modelling the shear behaviour of rock joints with asperity damage under constant normal stiffness, Rock Mech. Rock Eng. 48(1) (2015) 179-195.

43. N. Huang et al., Estimation of permeability of 3-D discrete fracture networks: An alternative possibility based on trace map analysis, Eng. Geol. 226 (2017) 12-19.

44. S. R. Brown, Fluid flow through rock joints: The effect of surface roughness, J. Geophys. Res. Solid Earth 92(B2) (1987) 1337-1347.

45. V. Vilarrasa et al., Shear-induced flow channels in a single rock fracture and their effect on solute transport, Transp. Porous Med. 87(2) (2011) 503-523.

46. R. Liu et al., Mathematical expressions for estimating equivalent permeability of rock fracture networks, Hydrogeol. J. 24(7) (2016) 1623-1649.

47. H. S. Lee and T. F. Cho, Hydraulic characteristics of rough fractures in linear flow under normal and shear load, Rock Mech. Rock Eng. 35(4) (2002) 299318.

48. M. K. Rahman, M. M. Hossain and S. S. Rahman, A shear-dilation-based model for evaluation of hydraulically stimulated naturally fractured reservoirs, Int. J. Numer. Anal. Met. 26(5) (2002) 469497. 
N. Huang et al.

49. B. Li et al., Experimental study of the hydromechanical behavior of rock joints using a parallelplate model containing contact areas and artificial fractures, Int. J. Rock Mech. Min. Sci. 45(3) (2008) $362-375$.
50. H. M. Kim and J. Inoue, Analytical approach for anisotropic permeability through a single rough rock joint under shear deformation, J. Geophys. Res. Solid Earth 108(B8) (2003) 2366. 